\title{
Synergy of BMI and family history on diabetes: the Humboldt Study
}

Yue Chen ${ }^{1, *}$, Donna C Rennie ${ }^{2,3}$ and James A Dosman ${ }^{2}$

'Department of Epidemiology and Community Medicine, Faculty of Medicine, University of Ottawa, 451 Smyth Road, Ottawa, Ontario, Canada, K1H 8M5: ${ }^{2}$ Institute of Agricultural Rural and Environmental Health, University of Saskatchewan, Saskatoon, Saskatchewan, Canada: ${ }^{3}$ College of Nursing, University of Saskatchewan, Saskatoon, Saskatchewan, Canada

Submitted 5 December 2008: Accepted 20 July 2009: First published online 26 August 2009

\begin{abstract}
Objective: To examine the joint effect of family history and BMI on diabetes.

Design: Cross-sectional study.

Setting: A rural community in Saskatchewan, Canada.

Subjects: The analysis was based on data from 2081 adults, 18-79 years of age, who participated in the Humboldt Study conducted in 2003. Doctor-diagnosed diabetes and family history of diabetes of biological parents and siblings were self-reported. Body weight and height were objectively measured. The interaction of family history and BMI on diabetes was assessed on an additive scale.

Results: The prevalence of diabetes was $7 \cdot 9 \%$, and BMI and history of diabetes were two important predictors. The adjusted prevalence ratios were $1.76(95 \% \mathrm{CI}$ $1 \cdot 37,2 \cdot 27)$ and $2 \cdot 59(95 \%$ CI $2 \cdot 05,3 \cdot 31)$ for those with a BMI of $25 \cdot 0-29 \cdot 9 \mathrm{~kg} / \mathrm{m}^{2}$ and of at least $30 \mathrm{~kg} / \mathrm{m}^{2}$, respectively, compared with a BMI of less than $25 \mathrm{~kg} / \mathrm{m}^{2}$, and was $2 \cdot 41(95 \%$ CI $2 \cdot 08,2 \cdot 80)$ for those with a family history of diabetes $v$. those without. The data indicated an additive interaction of family history and BMI on diabetes.

Conclusions: When exposed to both family history and overweight/obesity, individuals would have an increased risk that was greater than the sum of their single effects. Reduction of BMI would also reduce the risk of diabetes associated family history.
\end{abstract}

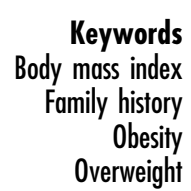

Keywords amily history Overweight
Type 2 diabetes has increased rapidly internationally. Type 2 diabetes is a complex disorder, and both genetic and environmental factors play an important role in the disease development. Family history of diabetes increases the risk of type 2 diabetes ${ }^{(1-6)}$. The more cases of diabetes found in a family, the younger the age of onset of type 2 diabetes $^{(7)}$. Family history of type 2 diabetes is associated with decreased insulin sensitivity and an impaired balance between insulin sensitivity and insulin secretion ${ }^{(8)}$. A number of genes have been identified to be associated with type 2 diabetes ${ }^{(9-11)}$.

The prevalence of type 2 diabetes is predicted to increase to epidemic proportions in the coming decades, primarily due to lifestyle changes ${ }^{(12)}$. A rapid increase in worldwide prevalence of obesity is believed to be a main reason ${ }^{(13)}$. The increase in diabetes prevalence over recent decades has disproportionately comprised persons with high levels of obesity ${ }^{(14-17)}$.

There is increasing evidence for interactions between obesity and genetic factors in the development of diabetes $^{(18)}$. Obesity modifies the effects of the Asp905Tyr variant of $P P P 1 R 3 A$ on risk of type 2 diabetes and insulin sensitivity ${ }^{(19)}$. Obesity is associated with type 2 diabetes only among individuals with high serum $\gamma$-glutamyltransferase (GGT) activity, not in those with low serum GGT ${ }^{(20)}$. A family history of type 2 diabetes is associated with lower sensitivity to activated protein $\mathrm{C}$ in overweight and obese premenopausal women ${ }^{(21)}$. Family history reflects genetic susceptibility in addition to other factors, e.g. common environmental exposures, and we hypothesise that a family history of diabetes modifies the association between obesity and type 2 diabetes and that overweight/obesity is more strongly associated with type 2 diabetes among those with a family history than those without a family history of the disease.

\section{Methods}

We conducted a cross-sectional study in the town of Humboldt, Saskatchewan, in 2003. The target population of the study was all the town residents 6 years of age or 
more and was conducted among children (6-17 years) and adults (18-79 years), separately; the present analysis was based on data from adult residents only. A total of 2090 adult residents, $18-79$ years of age (71\% of the target population), participated in the study, and the study methods have been detailed in earlier reports ${ }^{(22,23)}$. Briefly, canvassers contacted all households within the town and surrounding areas and asked all eligible adult subjects in each home to participate in the study and to complete a written consent of participation. A questionnaire left by the canvassers was completed in the home by each subject and returned during a pre-arranged clinic visit. Written informed consent was obtained from each participant. The present study was approved by the University of Saskatchewan Research Ethics Board.

Respondents who answered the following question affirmatively were considered as having diabetes: 'Has a doctor ever said you had diabetes?' Family history of diabetes was obtained by asking questions if their biological parents/sibling had diabetes. Weight was measured to the nearest $0.1 \mathrm{~kg}$ using a calibrated hospital spring scale with subjects dressed in normal indoor clothing without shoes. Height in centimetres was measured against a wall using a fixed tape measure with participants standing shoeless on a hard surface. BMI was calculated as weight $(\mathrm{kg}) /$ height $^{2}\left(\mathrm{~m}^{2}\right)$. Subjects were grouped into three categories based on BMI $\left(<25 \cdot 0,25 \cdot 0-29 \cdot 9\right.$ and $\left.\geq 30 \mathrm{~kg} / \mathrm{m}^{2}\right)$. Adults with a BMI of $25 \cdot 0-29 \cdot 9 \mathrm{~kg} / \mathrm{m}^{2}$ were considered to be overweight and those with a BMI $\geq 30 \mathrm{~kg} / \mathrm{m}^{2}$ were defined as being obese.

Participants in the low education category did not proceed beyond secondary school; the high education category included subjects admitted to college or university, as well as those with a post-secondary school certificate or diploma. Subjects were classified into low$(<\$ 50000)$ and high-income $(\geq \$ 50000)$ groups based on total household income. Current smokers were participants who reported smoking every day or almost everyday, and had smoked at least twenty packs during their lifetime. Ex-smokers were those who were regular smokers but, at the time of the survey, had quit for at least 6 months. A regular drinker was defined as a person who drank alcoholic beverages at least once per week, on average.

The relationships between BMI, family history and diabetes were examined among 2081 adults aged 18-79 years, who provided information on BMI, family history and diabetes. We calculated the prevalence of diabetes according to various risk factors. Log-binomial regression model was used to estimate unadjusted and adjusted prevalence ratio (PR) for associations among BMI, family history and diabetes ${ }^{(24)}$. The maximum likelihood estimations of PR were obtained and likelihood ratio CI were used for statistical inference. We also used a log-binomial regression model to evaluate the departures from additivity after adjustment for potential confounders. Indicator terms from each category of joint exposure were constructed in the model. People who were exposed neither to overweight/obesity nor to family history were defined as the reference category. Five indicator terms dealing with the joint effects of overweight/obesity and family history were built into the model. They indicate either the presence of each exposure and the absence of the other or the presence of joint exposure. We used the method developed by $\mathrm{Zou}^{(25)}$ to estimate the additive effect of family history and BMI on the prevalence of diabetes. Relative excess risk of interaction (RERI), attributable proportion of interaction and Rothman's synergy index were calculated to access deviation from the additive model $^{(25)}$.

\section{Results}

There were more female than male participants, $56 \% v$. $44 \%$. The participants had an average age of 51.9 (SD $15 \cdot 8$ ) years. The prevalence of doctor-diagnosed diabetes was $7 \cdot 9 \%$ in adults aged $18-79$ years. Table 1 shows the characteristics of the study population associated with the prevalence of diabetes. Age, socio-economic status, smoking and alcohol drinking were significantly associated with the crude prevalence of diabetes.

The prevalence increased significantly with BMI (Table 2). Of the participants, $23.0 \%$ of the participants reported that at least one of their parents had diabetes and $12.5 \%$ reported that at least one of their siblings had the disease. Overall, $29 \cdot 7 \%$ of the subjects had one or more diabetic parents or siblings. The prevalence of doctor-diagnosed diabetes was significantly higher among those with a family history of diabetes compared with those without (Table 2).

Table 1 Prevalence of diabetes according to characteristics of the study population 18-79 years of age, the Humboldt Study, 2003

\begin{tabular}{|c|c|c|c|c|}
\hline & $n$ & Cases & $\%$ & $\chi^{2}(P$ value $)$ \\
\hline \multicolumn{5}{|l|}{ Age (years) } \\
\hline $18-39$ & 513 & 11 & $2 \cdot 1$ & \multirow{3}{*}{$81 \cdot 23(<0.001)$} \\
\hline $40-59$ & 808 & 41 & $5 \cdot 1$ & \\
\hline 60-79 & 760 & 112 & $14 \cdot 7$ & \\
\hline \multicolumn{5}{|l|}{ Sex } \\
\hline Male & 906 & 77 & $8 \cdot 7$ & \multirow[t]{2}{*}{$0.84(0.358)$} \\
\hline Female & 1175 & 87 & $7 \cdot 4$ & \\
\hline \multicolumn{5}{|c|}{ Educational level } \\
\hline Low & 1113 & 103 & $9 \cdot 3$ & \multirow{3}{*}{$11.43(0.003)$} \\
\hline High & 931 & 55 & $5 \cdot 9$ & \\
\hline Unknown & 37 & 6 & $16 \cdot 7$ & \\
\hline \multicolumn{5}{|l|}{ Income } \\
\hline Low & 846 & 80 & $9 \cdot 5$ & \multirow{3}{*}{$9.91(0.007)$} \\
\hline High & 769 & 42 & $5 \cdot 5$ & \\
\hline Unknown & 466 & 42 & $9 \cdot 0$ & \\
\hline \multicolumn{5}{|l|}{ Smoking status } \\
\hline Non-smoker & 1103 & 77 & $7 \cdot 0$ & \multirow{3}{*}{$7 \cdot 29(0 \cdot 026)$} \\
\hline Ex-smoker & 719 & 72 & $10 \cdot 0$ & \\
\hline Smoker & 259 & 15 & $5 \cdot 8$ & \\
\hline \multicolumn{5}{|l|}{ Alcohol use } \\
\hline No & 826 & 99 & $12 \cdot 0$ & \multirow{3}{*}{$31 \cdot 81(<0.001)$} \\
\hline Yes & 1232 & 65 & $5 \cdot 2$ & \\
\hline Unknown & 23 & 1 & $4 \cdot 4$ & \\
\hline
\end{tabular}


Table 2 Prevalence of diabetes associated with BMI and family history in adults 18-79 years of age, the Humboldt Study, 2003

\begin{tabular}{lrrrr}
\hline & $n$ & Cases & $\%$ & $\chi^{2}(P$ value $)$ \\
\hline BMI $\left(\mathrm{kg} / \mathrm{m}^{2}\right)$ & & & & \\
$\quad<25$ & 565 & 20 & $3 \cdot 5$ & \\
$25 \cdot 0-29 \cdot 9$ & 779 & 55 & $7 \cdot 1$ & $33 \cdot 26(<0 \cdot 001)$ \\
$\quad \geq 30$ & 737 & 89 & $12 \cdot 1$ & \\
Parent with diabetes & & & & \\
$\quad$ No & 1603 & 97 & $6 \cdot 1$ & $32 \cdot 18(<0 \cdot 001)$ \\
$\quad$ Yes & 478 & 67 & $14 \cdot 0$ & \\
Sibling with diabetes & & & & \\
$\quad$ No & 1821 & 102 & $5 \cdot 6$ & $104 \cdot 32(<0 \cdot 001)$ \\
$\quad$ Yes & 260 & 62 & $23 \cdot 8$ & \\
Parent or sibling & & & & \\
$\quad$ with diabetes & & & & \\
$\quad$ No & 1462 & 71 & $4 \cdot 9$ & $61.93(<0 \cdot 001)$ \\
$\quad$ Yes & 619 & 93 & $15 \cdot 0$ & \\
\hline
\end{tabular}

Table 3 Unadjusted and adjusted ${ }^{\star}$ prevalence ratios (PR) and $95 \% \mathrm{Cl}$ for diabetes in relation to $\mathrm{BMI}$ and family history in adults 18-79 years of age, the Humboldt Study, 2003

\begin{tabular}{|c|c|c|c|c|}
\hline & \multicolumn{2}{|c|}{ Unadjusted } & \multicolumn{2}{|c|}{ Adjusted } \\
\hline & PR & $95 \% \mathrm{Cl}$ & PR & $95 \% \mathrm{Cl}$ \\
\hline \multicolumn{5}{|l|}{ BMI $\left(\mathrm{kg} / \mathrm{m}^{2}\right)$} \\
\hline$<25 \cdot 0$ & $1 \cdot 00$ & & $1 \cdot 00$ & \\
\hline $25 \cdot 0-29 \cdot 9$ & 1.99 & $1 \cdot 23,3 \cdot 37$ & $1 \cdot 76$ & $1 \cdot 37,2 \cdot 27$ \\
\hline$\geq 30 \cdot 0$ & $3 \cdot 41$ & $2 \cdot 17,5 \cdot 63$ & $2 \cdot 59$ & $2 \cdot 05,3 \cdot 31$ \\
\hline \multicolumn{5}{|c|}{ Family history of diabetes } \\
\hline Parent & $2 \cdot 32$ & $1 \cdot 72,3 \cdot 10$ & $2 \cdot 11$ & $1 \cdot 83,2 \cdot 44$ \\
\hline Sibling & $4 \cdot 26$ & $3 \cdot 18,5 \cdot 66$ & $2 \cdot 73$ & $2 \cdot 39,3 \cdot 11$ \\
\hline Parent or sibling & 3.09 & $2 \cdot 31,4 \cdot 17$ & $2 \cdot 41$ & $2 \cdot 08,2 \cdot 80$ \\
\hline
\end{tabular}

*Adjusted for age, alcohol drinking and family history for BMI associated with diabetes, and adjusted for age, alcohol drinking and BMI for family history associated with diabetes.

A logistic regression model was first utilised to assess the impact of BMI, family history and individual characteristics on diabetes. Non-significant variables including sex, education, income and smoking status were excluded from further analysis. A log-binomial regression model was then used to assess the independent effect of BMI and family history on the prevalence of doctor-diagnosed diabetes. After controlling for age, alcohol drinking and family history, the adjusted PR for those with a BMI of at least $30 \mathrm{~kg} / \mathrm{m}^{2}$ and a BMI of $25 \cdot 0-29 \cdot 9 \mathrm{~kg} / \mathrm{m}^{2}$, compared with a BMI of less than $25 \mathrm{~kg} / \mathrm{m}^{2}$, was $2 \cdot 59$ (95\% CI $\left.1 \cdot 05,3 \cdot 31\right)$ and $1 \cdot 76$ (95\% CI $1 \cdot 37,2 \cdot 27$ ), respectively (Table 3). After controlling for age, alcohol drinking and BMI, the adjusted PR was $2 \cdot 41(95 \%$ CI $2 \cdot 08,2 \cdot 80)$ for those with a family history compared with those without (Table 3 ).

Figure 1 shows that the prevalence of doctor-diagnosed diabetes increased with BMI more rapidly in those with a family history of diabetes than those without a family history. It seems that those with a family history are more susceptible to diabetes due to overweight/obesity.

A log-binomial regression model was used to evaluate the departures from additivity for BMI and family history associated with diabetes, after adjustment for age and

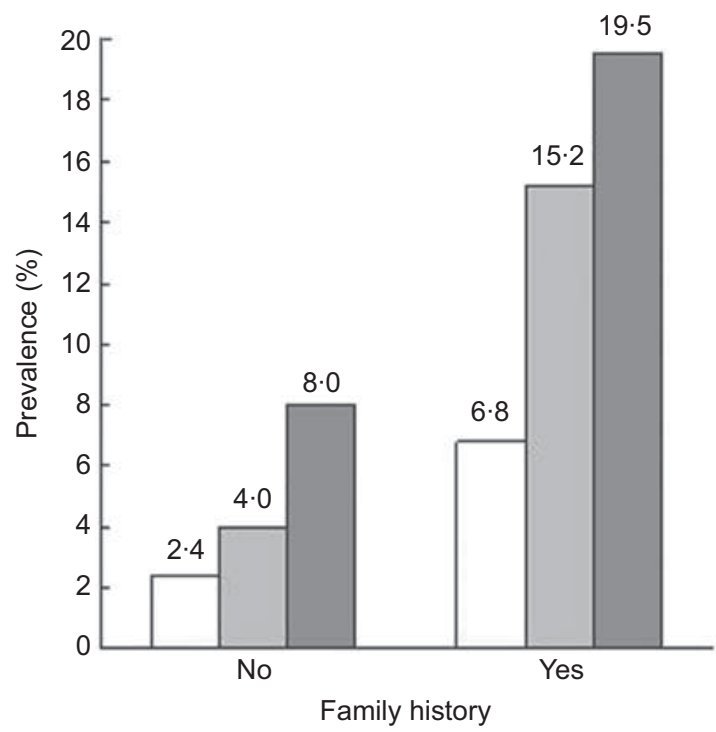

Fig. 1 Prevalence of diabetes by family history and BMI in adults aged $18-79$ years ( $\square$, normal; $\square$, overweight; $\square$, obese)

Table 4 Adjusted $^{*}$ prevalence ratios (PR) and $95 \% \mathrm{Cl}$ for the combined effects of BMI and family history on diabetes in adults 18-79 years of age, the Humboldt Study, 2003

\begin{tabular}{|c|c|c|c|c|}
\hline \multirow[b]{3}{*}{ BMI $\left(\mathrm{kg} / \mathrm{m}^{2}\right)$} & \multicolumn{4}{|c|}{ Parent or sibling with diabetes } \\
\hline & \multicolumn{2}{|c|}{ No } & \multicolumn{2}{|c|}{ Yes } \\
\hline & PR & $95 \% \mathrm{Cl}$ & PR & $95 \% \mathrm{Cl}$ \\
\hline$<25 \cdot 0$ & $1 \cdot 00$ & & $2 \cdot 29$ & $1 \cdot 45,3 \cdot 62$ \\
\hline $25 \cdot 0-29 \cdot 9$ & $1 \cdot 43$ & $0 \cdot 97,2 \cdot 19$ & $4 \cdot 73$ & $3 \cdot 24,6 \cdot 94$ \\
\hline$\geq 30 \cdot 0$ & $2 \cdot 83$ & $2 \cdot 01,4 \cdot 14$ & $5 \cdot 70$ & $3 \cdot 98,8 \cdot 17$ \\
\hline
\end{tabular}

*Adjusted for age and alcohol drinking.

alcohol drinking. The risk of diabetes was approximately three-fold higher for those who either had family history or were obese before adjustment (Table 4). For those who had both family history and obesity, the PR was 5.70 (95\% CI 3.98, 8·17; Table 4). The RERI was $1 \cdot 92$, and $85 \%$ of the diabetes risk was attributed to the synergy between overweight/obesity and family history. The combined effect of overweight/obesity and family history accounted for 36\% of those subjects who developed diabetes.

\section{Discussion}

Obesity is a major determinant of type 2 diabetes, and a number of studies have confirmed the association between obesity and type 2 diabetes $^{(26)}$. BMI, waist circumference and waist-to-hip ratio are all associated with type 2 diabetes ${ }^{(27)}$. Based on data from thirty-two studies, the pooled relative risks for incident diabetes were $1 \cdot 87$ (95\% CI $1 \cdot 67,2 \cdot 10), 1 \cdot 87$ (95\% CI $1 \cdot 58,2 \cdot 20)$ and $1 \cdot 88(95 \%$ CI $1 \cdot 61,2 \cdot 19)$ per SD of BMI, waist circumference and waist-to-hip ratio ${ }^{(27)}$. A combined analysis of twentyseven studies from the Asia Pacific region, which included 
154989 subjects with 1244793 person-years of follow-up, has shown that each $2 \mathrm{~kg} / \mathrm{m}^{2}$ reduction of BMI is associated with a $27 \%(23-30 \%)$ lower risk of diabetes ${ }^{(28)}$. Lost weight after gastric bypass surgery reduces the risk of diabetes ${ }^{(26)}$.

It is clear that obesity is associated with an increased risk of developing insulin resistance and type 2 diabetes. In obese individuals, adipose tissue releases increased amounts of NEFA, glycerol, hormones, adiponectin, resistin, TNF- $\alpha$ and IL- 6 , and results in an impairment of insulin sensitivity, development of insulin resistance and dysfunction of pancreatic islet $\beta$-cells ${ }^{(15,16)}$.

Family history of diabetes has been recognised as an important risk factor for diabetes. Family history of diabetes is significantly associated with the risk of diabetes $^{(2,29)}$ and has an exponential impact on ageingassociated increase in the risk of diabetes ${ }^{(30)}$. Our study indicated that family history was associated with a higher risk of diabetes in adults 18-79 years of age. Siblings' status of diabetes tended to have a greater impact on the prevalence of diabetes than that of parents, but the difference was not statistically significant. They were merged in the analysis of interaction with overweight/obesity.

It has been found that declines in insulin sensitivity and in the disposition index, and increases in fasting glucose are significantly influenced by a maternal history of type 2 diabetes $^{(31)}$. In children, family history of type 2 diabetes is associated with decreased insulin sensitivity and clearance $^{(8)}$.

Although there is strong evidence for both family history and obesity being important determinants of type 2 diabetes, it has not been well understood if family history and obesity synergistically influence diabetes. In an earlier cross-sectional study of 3128 men and 4821 women aged 35-56 years, combined exposure to a family history of diabetes and lifestyle-related risk factors, including obesity, had a greater effect on type 2 diabetes than any of these factors alone. The additive interaction was statistically significant for a family history of diabetes and obesity in women with prediabetes, but not in others ${ }^{(4)}$. In our study, we asked questions about family history of their biological father, mother and siblings and one-third of the study population had at least one of the relatives having the disease. The synergistic effect of family history and overweight/obesity was statistically significant, indicated by the indices of synergism.

The mechanisms for possible synergistic influence of a family history of diabetes and obesity on prevalent type 2 diabetes need to be further explored. Family history scores based on the diabetes status of the participants' parents and older siblings were found to be significantly correlated with reduced insulin sensitivity and increased subcutaneous and visceral fat in families from San Antonio and Los Angeles, but not in the leaner Hispanic families from San Luis Valley ${ }^{(32)}$. The authors of the study believed that the lack of an association between family history score and insulin resistance/fat accumulation in leaner families is likely due to a suppression of the expression of transmitted diabetes genes in leaner, more physically active populations ${ }^{(32)}$. If this speculation is true, it may explain our observation that family history has a larger influence on diabetes in the overweight/ obese than normal weight populations.

There are several limitations for the study. First, it is a cross-sectional study and we are not able to make any firm inclusion about causality. Second, the study is relatively small and only limited variables are included in the log-binomial regression model. This approach allowed us to produce PR estimates and to provide more accurate estimates for BMI and family history synergism. Third, the diagnosis of diabetes was self-reported, although validated questions were used in the survey.

Since type 2 diabetes poses substantial burdens of medical care and financial resources for individual patients, their families and society as a whole, prevention and control of the disease is of great importance. Family history represents valuable information on genetic and common environmental exposures. Family history can be a useful screening tool to identify people at high risk for diabetes $^{(5,29,33)}$ and is considered as a promising public health tool to fight the growing epidemic of diabetes ${ }^{(34)}$. The validity and utility of using family history as a screening tool can be largely improved by combining with BMI or other measures of adiposity, which will help in identifying high-risk groups and developing targeted intervention programmes.

\section{Acknowledgements}

The present study was supported by a grant from the Canadian Institutes of Health Research (200203MOP100752-POP-CCAA-11829). None of the authors had a personal or financial conflict of interest with any aspect of this research. All authors contributed to the conception and design of the study; D.C.R. and J.A.D. supervised the data collection; Y.C. performed the statistical analysis; and all authors contributed to the writing of the manuscript.

\section{References}

1. Carlsson S, Midthjell K \& Grill V (2007) Influence of family history of diabetes on incidence and prevalence of latent autoimmune diabetes of the adult: results from the NordTrondelag Health Study. Diabetes Care 30, 3040-3045.

2. Valdez R, Yoon PW, Liu T \& Khoury MJ (2007) Family history and prevalence of diabetes in the US population: the 6-year results from the National Health and Nutrition Examination Survey (1999-2004). Diabetes Care 30, 2517-2522.

3. Yeung EH, Pankow JS, Astor BC, Powe NR, Saudek CD \& Kao WH (2007) Increased risk of type 2 diabetes from a family history of coronary heart disease and type 2 diabetes. Diabetes Care 30, 154-156.

4. Hilding A, Eriksson AK, Agardh EE, Grill V, Ahlbom A, Efendic S \& Ostenson CG (2006) The impact of family history of diabetes and lifestyle factors on abnormal glucose 
regulation in middle-aged Swedish men and women. Diabetologia 49, 2589-2598.

5. Hariri S, Yoon PW, Moonesinghe R, Valdez R \& Khoury MJ (2006) Evaluation of family history as a risk factor and screening tool for detecting undiagnosed diabetes in a nationally representative survey population. Genet Med $\mathbf{8}$, 752-759.

6. Bjornholt JV, Erikssen G, Liestol K, Jervell J, Thaulow E \& Erikssen J (2000) Type 2 diabetes and maternal family history: an impact beyond slow glucose removal rate and fasting hyperglycemia in low-risk individuals? Results from 22.5 years of follow-up of healthy nondiabetic men. Diabetes Care 23, 1255-1259.

7. Molyneaux L, Constantino M \& Yue D (2004) Strong family history predicts a younger age of onset for subjects diagnosed with type 2 diabetes. Diabetes Obes Metab 6 , 187-194.

8. Arslanian SA, Bacha F, Saad R \& Gungor N (2005) Family history of type 2 diabetes is associated with decreased insulin sensitivity and an impaired balance between insulin sensitivity and insulin secretion in white youth. Diabetes Care 28, 115-119.

9. Jafar-Mohammadi B \& McCarthy MI (2008) Genetics of type 2 diabetes mellitus and obesity: a review. Ann Med 40, 2-10.

10. Permutt MA, Wasson J \& Cox N (2005) Genetic epidemiology of diabetes. J Clin Invest 115, 1431-1439.

11. Das UN \& Rao AA (2007) Gene expression profile in obesity and type 2 diabetes mellitus. Lipids Health Dis 6, 35.

12. Gable D, Sanderson SC \& Humphries SE (2007) Genotypes, obesity and type 2 diabetes - can genetic information motivate weight loss? A review. Clin Chem Lab Med 45, 301-308.

13. Lorenzo C, Serrano-Rios M, Martinez-Larrad MT, GonzalezVillalpando C, Williams K, Gabriel R, Stem MP \& Haffner SM (2007) Which obesity index best explains prevalence differences in type 2 diabetes mellitus? Obesity (Silver Spring) 15, 1294-1301.

14. Gregg EW, Cheng YJ, Narayan KM, Thompson TJ \& Williamson DF (2007) The relative contributions of different levels of overweight and obesity to the increased prevalence of diabetes in the United States: 1976-2004. Prev Med 45, 348-352.

15. Kahn SE, Hull RL \& Utzschneider KM (2006) Mechanisms linking obesity to insulin resistance and type 2 diabetes. Nature 444, 840-846.

16. Keller U (2006) From obesity to diabetes. Int J Vitam Nutr Res 76, 172-177.

17. Smyth S \& Heron A (2006) Diabetes and obesity: the twin epidemics. Nat Med 12, 75-80.

18. Johnson RL, Williams SM \& Spruill IJ (2006) Genomics, nutrition, obesity, and diabetes. J Nurs Scholarsh 38, 11-18.

19. Mammarella S, Creati B, Staniscia T et al. (2007) Obesity modifies the effects of the Asp905Tyr variant of $P P P 1 R 3 A$ on risk of type 2 diabetes and insulin sensitivity. Diabetes Obes Metab 9, 759-761.

20. Lim JS, Lee DH, Park JY, Jin SH \& Jacobs DR Jr (2007) A strong interaction between serum $\boldsymbol{\gamma}$-glutamyltransferase and obesity on the risk of prevalent type 2 diabetes: results from the Third National Health and Nutrition Examination Survey. Clin Chem 53, 1092-1098.

21. Pannacciulli N, De Mitrio V, Sciaraffia M, Giorgino R \& De Pergola G (2001) A family history of type 2 diabetes is associated with lower sensitivity to activated protein $\mathrm{C}$ in overweight and obese premenopausal women. Thromb Haemost 86, 1593-1594.

22. Chen Y, Rennie D, Cormier Y \& Dosman J (2005) Sex specificity of asthma associated with objectively measured body mass index and waist circumference: the Humboldt Study. Chest 128, 3048-3054.

23. Chen Y, Rennie D, Cormier YF \& Dosman J (2007) Waist circumference is associated with pulmonary function in normal-weight, overweight, and obese subjects. Am J Clin Nutr 85, 35-39.

24. Deddens JA \& Petersen MR (2008) Approaches for estimating prevalence ratios. Occup Environ Med 65, 481, 501-506.

25. Zou GY (2008) On the estimation of additive interaction by use of the four-by-two table and beyond. $A m J$ Epidemiol 168, 212-224.

26. Wild SH \& Byrne CD (2006) ABC of obesity. Risk factors for diabetes and coronary heart disease. BMJ 333, 1009-1011.

27. Vazquez G, Duval S, Jacobs DR Jr \& Silventoinen K (2007) Comparison of body mass index, waist circumference, and waist/hip ratio in predicting incident diabetes: a metaanalysis. Epidemiol Rev 29, 115-128.

28. Ni Mhurchu C, Parag V, Nakamura M, Patel A, Rodgers A \& Lam TH (2006) Body mass index and risk of diabetes mellitus in the Asia-Pacific region. Asia Pac J Clin Nutr 15, $127-133$.

29. Annis AM, Caulder MS, Cook ML \& Duquette D (2005) Family history, diabetes, and other demographic and risk factors among participants of the National Health and Nutrition Examination Survey 1999-2002. Prev Chronic Dis 2, A19.

30. Shirakawa T, Ozono R, Kasagi F, Oshima T, Kamada N \& Kambe M (2006) Differential impact of family history on ageassociated increase in the prevalence of hypertension and diabetes in male Japanese workers. Hypertens Res 29, 81-87.

31. Kelly LA, Lane CJ, Weigensberg MJ, Koebnick C, Roberts CK, Davis JN, Toledo-Corral CM, Shaibi GQ \& Goran MI (2007) Parental history and risk of type 2 diabetes in overweight Latino adolescents: a longitudinal analysis. Diabetes Care 30, 2700-2705.

32. Mitchell BD, Zaccaro D, Wagenknecht LE, Scherzinger AL, Bergman RN, Haffner SM, Hokanson J, Norris JM, Rott JI \& Saad MF (2004) Insulin sensitivity, body fat distribution, and family diabetes history: the IRAS Family Study. Obes Res 12, 831-839.

33. Hariri S, Yoon PW, Qureshi N, Valdez R, Scheuner MT \& Khoury MJ (2006) Family history of type 2 diabetes: a population-based screening tool for prevention? Genet Med $\mathbf{8}, 102-108$.

34. Harrison TA, Hindorff LA, Kim H, Wines RC, Bowen DJ, McGrath BB \& Edwards KL (2003) Family history of diabetes as a potential public health tool. Am J Prev Med 24, 152-159. 